\title{
CT-guided fine-needle aspiration cytology of solid pseudopapillary tumor of the pancreas: Case series and review of literature
}

\author{
Anadi Roy Choudhury, Bhaskar Mitra, Palash Bhattacharya, \\ Aditi Bhattacharya
}

\begin{abstract}
Introduction: Solid pseudopapillary tumor is a rare entity considered to be a tumor of low malignant potential and therefore proper evaluation and diagnosis is important. Case Series: We report two cases of solid pseudopapillary tumor with special emphasis on cytomorphology, histopathology and immunohistochemistry. Preoperative image guided cytopathology is helpful for correct identification of this unusual neoplasm as complete resection is the treatment of choice and is associated with long term survival even in the presence of metastatic disease. Conclusion: Solid pseudopapillary tumor is a distinctive pancreatic neoplasm whose cell phenotype remains a mystery, so we report this case series with extensive literature review.
\end{abstract}

Keywords: Fine-needle aspiration cytology, Solid pseudopapillary tumor, Pancreas

$$
* * * * * * * * *
$$

Choudhury AR, Mitra B, Bhattacharya P, Bhattacharya A. CT-guided fine-needle aspiration cytology of solid

Anadi Roy Choudhury ${ }^{1}$, Bhaskar Mitra $^{2}$, Palash Bhattacharya $^{1}$, Aditi Bhattacharya ${ }^{1}$

Affiliations: ${ }^{1}$ Assistant Professor, Department of Pathology, RG Kar Medical College \& Hospital, Kolkata, West-Bengal, India; ${ }^{2}$ Assistant Professor, Department of Pathology, Midnapore Medical College \& Hospital, Paschim Medinipur, West-Bengal, India.

Corresponding Author: Dr. Bhaskar Mitra, 54/2/G, Feeder Road, P.O- Belgharia, Pin Code - 700056. Kolkata, WestBengal, India; Ph: +91 9874174040; Email: bhaskarmitra12@gmail.com

Published: 9 July 2012 pseudopapillary tumor of the pancreas: Case series and review of literature. International Journal of Hepatobiliary and Pancreatic Diseases 2012;2:15-18.

Article ID: 100007IJHPDARC2012

$$
* * * * * * * * *
$$

doi:10.5348/ijhpd-2012-7-CS-5

\section{INTRODUCTION}

Solid pseudopapillary tumor of the pancreas (SPTP) is a rare neoplasm with a reported frequency of $0.17-2.7 \%$ of all nonendocrine tumors of the pancreas [1], and $6.5 \%$ of all pancreatic tumors and tumor-like lesions resected as reported in one large institutional study [2]. It was first described by Frantz et al. and it was also called papillary and solid epithelial neoplasm, papillary-cystic neoplasm and cystic-solid papillary carcinoma until the WHO pancreatic tumor working group termed it as Solid pseudopapillary neoplasm [3].

Though rare it can be diagnosed accurately by Computed Tomography (CT)-guided fine needle aspiration cytology preoperatively as there are some other pancreatic tumors that share the same CT findings of encapsulated and cystic tumor like SPTP. Cytologically the features of SPTP are distinct from these tumors. It is important that this tumor be accurately diagnosed because management protocols differ from other tumor types originating in the pancreas.

\section{CASE REPORT}

\section{Case 1}

A 20-year-old woman was admitted with complaints of an unexplained, episodic, epigastric pain for six months in the department of medicine. Clinical and 
laboratory examinations revealed mild splenomegaly and low serum amylase and carcinoembryonic antigen (CEA) levels. CT-scan revealed an encapsulated mass of $8.2 \times 7.5 \mathrm{~cm}$ in the pancreatic tail with solid and cystic consistency and adjacent spleen was not invaded by the tumor. CT-guided fine needle aspiration was performed using 22-gauge spinocain needles via a trans-gastric approach. The aspirated material was smeared onto glass slides, air-dried, and stained with Leishman and Giemsa stain for preliminary diagnostic interpretation. Other smears also were fixed immediately in 95\% alcohol for subsequent Papanicolaou staining. Patient was then transferred to the Department of Surgery for definitive therapy. Distal pancreatectomy and splenectomy was performed and the specimen was sent to our department.

\section{Case 2}

A 19-year-old female presented to the out-patient department of general surgery with complaints of dragging sensation in the upper part of the abdomen with epigastric tenderness. Upper GI endoscopy was within normal limits. CT scan showed an $8.34 \times 7.22 \mathrm{~cm}$ well encapsulated, complex, heterogeneous mass with cystic and solid components in the head of the pancreas without evidence of invasion (Figure 1). CT-guided FNAC was performed. Subsequently, the patient was admitted in the department of Gastroenterology and operated with Whipple' operation and the specimen sent to our department for confirmation of the diagnosis and extent of invasion.

Cytology smears showed moderate cellularity with cells arranged in loosely cohesive clusters and in papillary fashion. Individual cells were uniform and bland with round to oval nuclei having evenly dispersed or somewhat granular chromatin and small indistinct nucleoli. Mitotic figures and nuclear atypia were absent. The cytoplasm ranged from scant to moderate (Figure $2 \mathrm{~A}, \mathrm{~B}$ ).

Grossly, both tumours were well circumscribed globular mass with cystic variegated areas on cut section. Adjacent spleen in the first case and duodenum in the second case were not involved.

Histologically, the tumor cells were arranged in solid sheets, cords and pseudopapillae. Individual cells were uniform, medium sized, round with eosinophilic cytoplasm and bland nuclei with finely dispersed chromatin and inconspicuous nucleoli and some nuclei showed indentation. Mitotic figures were scanty. Vascular invasion was not noted. Surrounding capsule showed calcification (Figure 3). CD10 immunostain was done which showed focal positivity of moderate intensity. With these classic features a diagnosis of SPTP was offered.

\section{DISCUSSION}

SPTP is a rare tumour occurring predominantly in adolescent girls and young women (mean age 35 years; range 8-67 years). It is rare in men (mean age 35 years; range $25-72$ years) without any apparent ethnic preference [3].

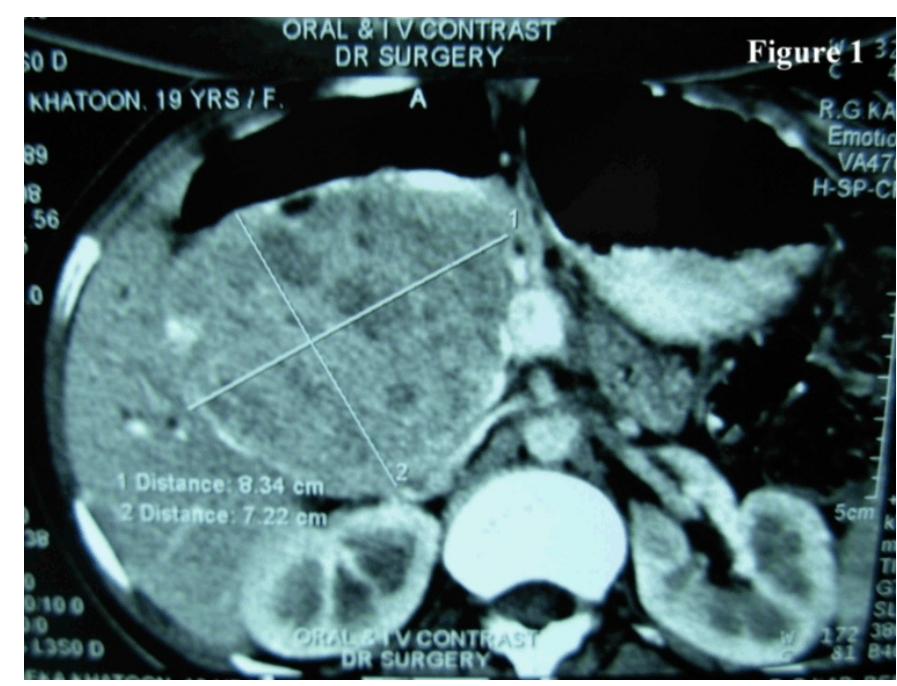

Figure 1: CT scan showing encapsulated, complex, heterogeneous mass with cystic and solid components in the head of the pancreas.

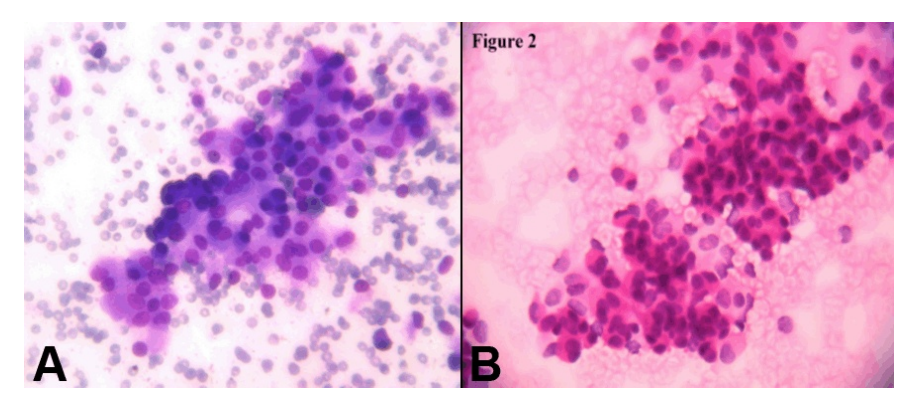

Figure 2: A) Leishman-Giemsa, and B) Pappanicolau stained smears showing cells arranged in loosely cohesive clusters (x400).

Clinically, SPTP may present as an abdominal mass with discomfort or pain or it may be an incidental finding in work up for unrelated conditions. Jaundice is rare even if the tumor arises from the head of the pancreas [3]. These tumors are generally large with a mean diameter of $10.3 \mathrm{~cm}$ and approximately $72 \%$ arise in the body and tail of the pancreas and less frequently in the head. SPTP may present at extrapancreatic sites such as mesocolon, retroperitoneum, omentum, liver and duodenum [4].

In our case, the sex (female) and age (20 years and 19 years respectively) and tumor localization (tail in the first case and body in the second case) were typical of SPTP.

SPTP usually runs a benign course though about $15 \%$ cases it may present with local extension into blood vessels and organs, local recurrence and distant metastasis [5]. Most of the tumors are benign appearing but tumors which have the potential to metastasize show cellular atypia. Pathologic features related to metastatic potential include diffuse growth pattern, venous invasion, nuclear pleomorphism, mitotic rate, necrosis and areas of dedifferentiation [6]. 


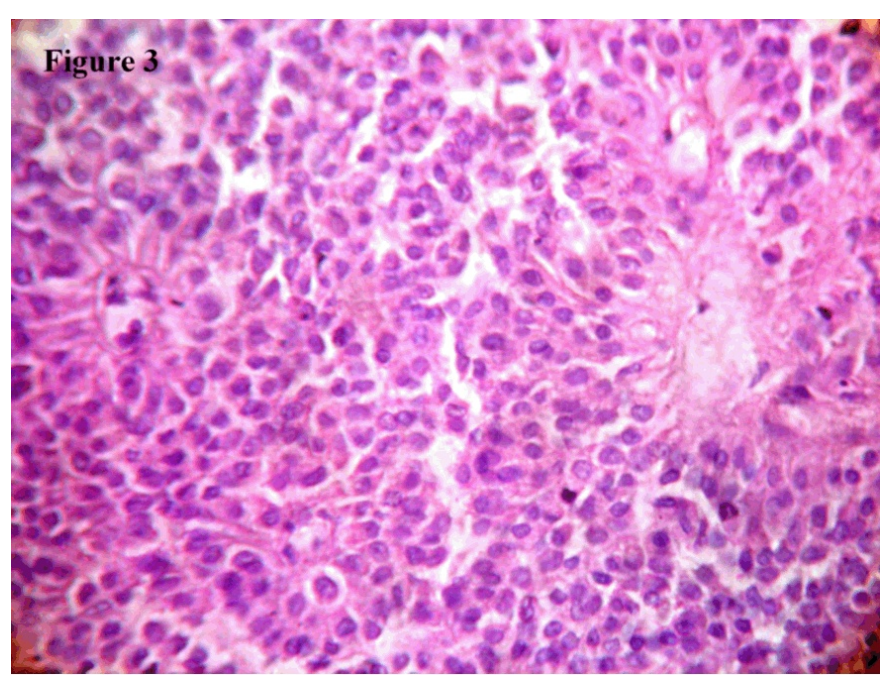

Figure 3: Histopathology section showing tumor cells arranged in solid sheets, cords and pseudopapillae. Individual cells were uniform, medium sized, round with eosinophilic cytoplasm and bland nuclei (H\&E, x400).

Ultrasonography (USG) and computed tomography (CT) scan reveal a sharply demarcated, variably solid and cystic mass without any internal septation. The tumor margin may contain calcifications.

Cytogically, it presents with highly cellular aspirates with single cells, loose clusters, and branching papillary fronds. Tumor cell are monotonous and bland with or without clefted nuclei (or nuclear grooves). Myxoid and metachromatic stroma and background material may be found. Necrotic debris is seen rarely when areas of cystic degeneration are sampled [7].

In our cases we did not get the necrotic material in the background. Immunohistochemistry of SPTP has produced conflicting results with regard to tumor cell phenotyping. Patil et al. suggested that SPTP possibly originate from the primordial cells and lack definite exocrine and endocrine differentiation [8]. Notohara et al. and others performed immunohistochemical analysis of 19 cases of SPTPs and showed that all of them exhibited immunoreactivity for CD56 and CD10. Fifteen of them expressed other neuroendocrine markers focally with the exception of Chromogranin A [9]. Another study of three SPTP cases demonstrated immunoreactivity for vimentin, CD10, CD56, synaptophysin and nuclear accumulation of beta catenin [10].

But due to financial constraints we have only done CD10 to confirm our diagnosis and found it to be positive.

\section{CONCLUSION}

A high index of suspicion is necessary to diagnose SPTP. In such cases CT-guided FNAC appears to be of value in preoperative diagnosis. Surgical excision offers the best chance for cure and patients have an excellent prognosis.

$$
* * * * * * * * *
$$

\section{Author Contributions}

Anadi Roy Choudhury - Substantial contributions to conception and design, Acquisition of data, Drafting the article, revising it critically for important intellectual content, Final approval of the version to be published Bhaskar Mitra - Substantial contributions to conception and design, Analysis and interpretation of data, Interpretation of Immunohistochemistry data, Drafting the article, Critical revision of article, Final approval of the version to be published

Palash Bhattacharya - Substantial contributions to conception and design, Drafting the article, Revising it critically for important intellectual content, Final approval of the version to be published

Aditi Bhattacharya - Substantial contributions to conception and design, Analysis and interpretation of data, Drafting the article, Revising it critically for important intellectual content, Final approval of the version to be published.

\section{Guarantor}

The corresponding author is the guarantor of submission.

\section{Conflict of Interest}

Authors declare no conflict of interest.

\section{Copyright}

(C) Anadi Roy Choudhury et al. 2012; This article is distributed under the terms of Creative Commons attribution 3.0 License which permits unrestricted use, distribution and reproduction in any means provided the original authors and original publisher are properly credited. (Please see www.ijhpd.com/copyrightpolicy.php for more information.)

\section{REFERENCES}

1. Pettinato G, Manivel JC, Ravetto C, et al. Papillary cystic tumor of the pancreas. A clinicopathologic study of 20 cases with cytologic, immunohistochemical, ultrastructural, and fl ow cytometric observations, and a review of the literature. Am J Clin Pathol 1992;98:478-8.

2. Kosmahl M, Pauser U, Peters K, et al. Cystic neoplasms of the pancreas and tumorlike lesions with cystic features: a review of 418 cases and a classification proposal. Virchows Arch 2004;445:168-78.

3. Hamilton SR, Aaltonen LA (Eds.). World Health Organization Classification of Tumours. Pathology and Genetics of Tumours of the Digestive System. IARC Press: Lyon 2000;246-8.

4. Salla C, Chatzipantelis P, Konstantinou P, Karoumpalis I, Pantazopoulou A, Dappola V. 
Endoscopic ultrasound-guided fine-needle aspiration cytology diagnosis of solid pseudopapillary tumor of the pancreas: A case report and literature review. World J Gastroenterol 2007;13(38):5158-63.

5. Pasquiou C, Scoazec JY, Gentil-Perret A, et al. Solid seudopapillary tumors of the pancreas. Pathology report of 13 cases. Gastroenterol Clin Biol 1999;23:207-14.

6. Nishihara K, Nagoshi M, Tsuneyoshi M, Yamaguchi K, Hayashi I. Papillary cystic tumor of pancreas: Assessment of their malignant potential. Cancer 1993;71:82-92.

7. Chhieng DC, Stelow EB. Pancreatic Cytopathology. 3rd Ed. New York: Springer 2007:95-107.

8. Patil TB, Shrikhande SV, Kanhere HA, Saoji R, Ramadwar M, Shukla PJ. Solid pseudopapillary neoplasm of the pancreas: a single institution experience of 14 cases. HPB (Oxford) 2006;8(2):148-50.

9. Notohara K, Hamazaki S, Tsukayama C, et al. SolidPseudopapillary Tumor of the Pancreas: Immunohistochemical Localization of Neuroendocrine Markers and CD10. Am J Surg Pathol 2000;24:1361-71.

10. Albores-Saavedra J, Simpson KW, Bilello SJ. The Clear Cell Variant of Solid Pseudopapillary Tumor of the Pancreas: A Previously Unrecognized Pancreatic Neoplasm. Am J Surg Pathol 2006;30:1237-42. 